\title{
CARACTERIZACIÓN PARA LA UBICACIÓN EN LA CAPTURA DE VIDEO APLICADO A TÉCNICAS DE VISIÓN ARTIFICIAL EN LA DETECCIÓN DE PERSONAS
}

\section{CHARACTERIZATION FOR LOCATION IN THE CAPTURE OF VIDEO APPLIED TO ARTIFICIAL VISION TECHNIQUES IN THE PEOPLE DETECTION}

\author{
Ing. Carlos V. Niño Rondón, MSc. Sergio A. Castro Casadiego, \\ PhD. Byron Medina Delgado
}

\author{
Universidad Francisco de Paula Santander, Facultad de Ingeniería. \\ Grupo de investigación y desarrollo en Electrónica y Telecomunicaciones (GIDET). \\ Av. Gran Colombia N ${ }^{\circ}$ 12E-96, San José de Cúcuta, Norte de Santander, Colombia. \\ Tel.: 57-7-5776655, Ext. 202 \\ E-mail: \{carlosvicentenr, sergio.castroc, byronmedina\}@ufps.edu.co
}

Resumen: Este documento muestra los resultados de la caracterización para la ubicación del dispositivo de captura de video para implementar en Python las técnicas de visión artificial de sustracción de fondo, detectores en cascada y flujo óptico, utilizadas en la detección de personas. Se confrontaron aspectos como altura y ángulo de inclinación del dispositivo, obteniendo una exactitud de $98.34 \%$ para la técnica de sustracción de fondo, con la cámara ubicada a 4 metros de altura con inclinación de $50^{\circ}$, siendo la de mejor rendimiento en el número de detecciones. En promedio, las pruebas realizadas a 4 metros presentan rendimiento de $87.96 \%$, a 8 metros, $88.19 \%$, a $50^{\circ}, 87.94 \%$ y a $90^{\circ}$ el rendimiento es de $88.19 \%$.

Palabras clave: Caracterización, dispositivo de captura de video, sustracción de fondo, detectores en cascada, flujo óptico.

\begin{abstract}
This document shows the results obtained during the characterization for the location of video capture device to implement in Python the techniques of artificial vision of background subtraction, cascade detectors and optical flow, used in the people detection. Aspects such as height and angle of inclination of the device were compared, obtaining an accuracy of $98.34 \%$ for the technique of subtraction of bottom, with the camera located at 4 meters high with an inclination of $50^{\circ}$, being the best performance in the number of detections. On average, tests carried out at 4 meters show a yield of $87.96 \%$, at 8 meters $88.19 \%$, at $50^{\circ}, 87.94 \%$ and at $90^{\circ}$ the yield is $88.19 \%$.
\end{abstract}

Keywords: Characterization, video capture device, background subtraction, cascade detectors, optical flow. 


\section{INTRODUCCIÓN}

La visión artificial hace referencia al conjunto de técnicas que permiten obtener cierta información y simular las capacidades de las personas para capturar una imagen o un vídeo, analizarlos y tomar decisiones en consecuencia (García S \& Caranqui S, 2015).

Igualmente, la visión artificial se presenta como una alternativa en los procesos de localización de objetos y personas, así como en la extracción de características correspondientes a las actividades realizadas por los humanos. (Leo, Medioni, Trivedi, Kanade, \& Farinella, 2017).

Actualmente, la detección de personas es uno de los temas de gran relevancia en los procesos de investigación (Franco, Ospina, Cuevas, \& Capacho, 2017). El propósito de estos procesos es identificar y monitorear las personas presentes en el cuadro de video analizado con aplicabilidad en video vigilancia y conteo automático de personas (Kajabad \& Ivanov, 2019).

El rendimiento de las técnicas de visión por computador depende de la calidad de la imagen de video suministrada al sistema de procesamiento, por lo que es común que aparezcan fallas al implementar este tipo de tecnología en espacios abiertos, ya que se presentan factores como cambios en la luminosidad, sombras y en algunos casos ausencia de luz. (Jeon et al., 2015).

En este artículo se propone determinar las consideraciones de ubicación óptimas para mejorar el resultado en el número de aciertos en la detección de personas para las técnicas de sustracción de fondo, detectores de cascada y flujo óptico. (Pérez, J, Castro, J 2018).

\section{METODOLOGÍA}

La metodología propuesta se basa en tres etapas: la toma de muestras de imágenes en diferentes condiciones de ubicación, la aplicación de las técnicas de detección de personas y la comparación con respecto al número de aciertos en las detecciones (Moctezuma-Ochoa, 2016).

\subsection{Consideraciones de ubicación}

La precisión de los procesos de detección humana depende tanto del rendimiento del dispositivo de captura de vídeo como de las características espaciales del lugar (Thacker et al., 2008).

Las características de ubicación que deben considerarse son: la altura de la ubicación y el ángulo de inclinación de la cámara, así como el nivel de luminosidad presente en la zona de captura de vídeo(Vidalia et al., 2019).

Los videos de prueba fueron tomados a alturas de 4 y 8 metros y los ángulos de inclinación del dispositivo de captura de video fueron $50^{\circ}$ y $90^{\circ}$ (Díaz, 2012). Asimismo, el nivel de luminosidad presente en el momento de la captura de dichos videos fue medido con el uso de un luxómetro digital. (Hernández Tolosa C, Contreras Eugenio B, Torres Sánchez C. 2016).

\subsection{Aplicación de las técnicas de detección}

La detección de personas tiene como objetivo distinguir entre los objetos en movimiento en una imagen de vídeo para su posterior clasificación (Paul, Haque, \& Chakraborty, 2013). En esta sección se estudian las técnicas de sustracción de fondo, detectores de cascada y flujo óptico y su codificación en el lenguaje de programación Python.

\subsubsection{Sustracción de fondo}

Detecta los cambios de posición de los objetos presentes en una imagen de vídeo. En términos generales, la técnica consiste en hacer una sustracción entre el fotograma de la imagen que se va a procesar y el fondo de la misma, obteniendo así el objeto presente en el fotograma de vídeo (Sobral \& Vacavant, 2014). En la tabla 1 , se muestran los procesos generales necesarios para la aplicación de la técnica de sustracción de fondo en Python.

\begin{tabular}{cc}
\hline Tabla 1. Algoritmo de sustracción de fondo \\
\hline 1: & definir valor en pixeles de ancho y alto \\
2: & ubicar una línea de prueba para conteo \\
3: & crear vector para las detecciones \\
4: & inicializar imagen de video \\
5: & inicializar el algoritmo de sustracción de \\
& fondo de mezclas gaussianas \\
6: & crear función para la posición de las \\
& detecciones \\
7: & mientras corre el video: \\
devolver el cuadro de video en curso & $\quad$ binarizar imagen de video \\
8: & aplicar filtros morfológicos \\
9: & definir umbral \\
10: & buscar contornos en la imagen \\
11: & analizar contornos \\
12: & validar contornos respecto a los \\
$13:$ & valores de ancho y alto \\
14: & dibujar rectángulo a las detecciones \\
& si los valores de la función están en el \\
$15:$ & vector de detecciones \\
\hline
\end{tabular}




\begin{tabular}{ll}
\hline 16: & \multicolumn{1}{c}{$+\mathbf{1}$ en el número de detecciones } \\
17: & pausa \\
18: & fin
\end{tabular}

La figura 1, muestra la aplicación de la técnica de sustracción de fondo en los videos de prueba tomados.

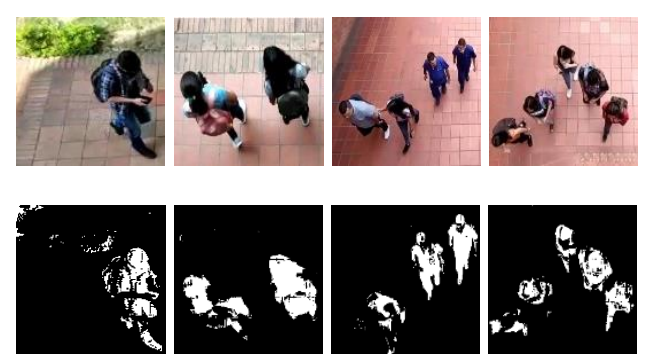

Fig. 1. Aplicación de la técnica de sustracción de fondo

\subsubsection{Detectores en cascada}

Acopla varios clasificadores con el fin de crear una estructura de varios niveles, acelerando así el proceso de detección. (Setjo, Achmad, \& Faridah, 2017).

La clasificación es el proceso más importante en un detector de cascada. Por eso se requiere un conjunto de datos entrenados para que el objeto en cuestión sea filtrado paso a paso por el clasificador, mejorando así los resultados de las detecciones (Mahkonen, Virtanen, \& Kämäräinen, 2018). En la tabla 2, se muestran los procesos generales necesarios para la aplicación de la técnica de detectores de cascada de cuerpo completo en Python.

\begin{tabular}{|c|c|}
\hline 1: & crear dataset \\
\hline 2: & entrenar dataset \\
\hline 3: & crear vector para las detecciones \\
\hline 4: & inicializar imagen de video \\
\hline 5: & inicializar clasificador en cascada \\
\hline 6: & $\begin{array}{l}\text { mientras corre el video: } \\
\text { devolver el cuadro de video en } \\
\text { curso }\end{array}$ \\
\hline 7: & binarizar imagen de video \\
\hline 8: & $\begin{array}{l}\text { buscar cuerpos completos } \\
\text { definir parámetros }\end{array}$ \\
\hline 9: & $\begin{array}{l}\text { dibujar rectángulo a las } \\
\text { detecciones }\end{array}$ \\
\hline 10: & $\begin{array}{l}\text { si los valores de la función estár } \\
\text { en el vector de detecciones }\end{array}$ \\
\hline 11: & $\begin{array}{l}\mathbf{+ 1} \text { en el número de } \\
\text { detecciones } \\
\text { pausa }\end{array}$ \\
\hline 12: & fin \\
\hline
\end{tabular}

En la figura 2, se observa la aplicación del algoritmo de detector en cascada de cuerpo completo para los videos de prueba capturados.
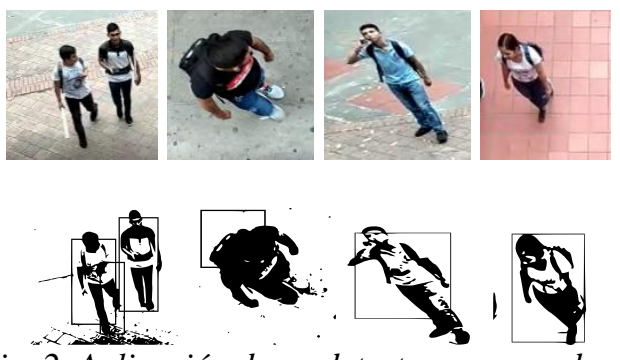

Fig. 2. Aplicación de un detector en cascada de cuerpo completo

\subsubsection{Flujo óptico}

Obtiene las estructuras correspondientes a la variación de las velocidades de los patrones de luminosidad en una imagen (Min \& Huang, 2016). Aunque permite obtener información relevante de los objetos, es susceptible variaciones en la luminosidad de la escena de vídeo(Sharmin \& Brad, 2012). En la tabla 3, se muestran los procesos generales requeridos para la aplicación de la técnica de flujo óptico en Python.

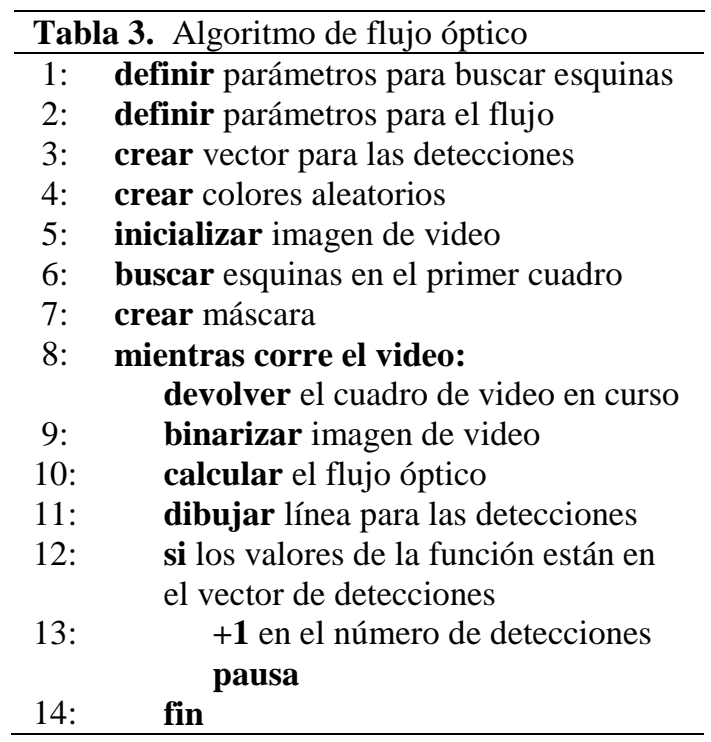

Así mismo, la figura 3 muestra la aplicación de la técnica de flujo óptico en los videos de prueba tomados.
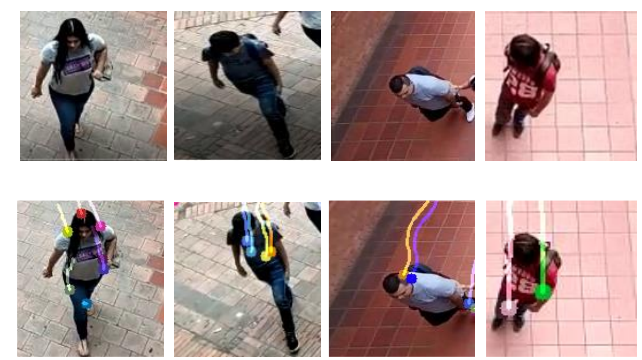
Fig. 3. Flujo óptico

vídeos utilizados en la aplicación de las técnicas de detección de personas.

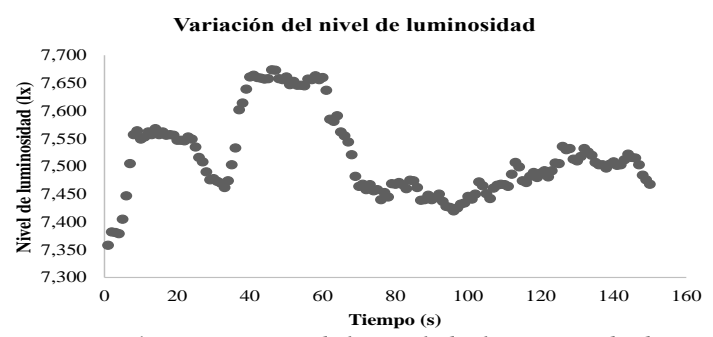

Fig. 4. Variación del nivel de luminosidad

El nivel de luminosidad obtenido fluctúa entre 7358 y 7674 luxes. Esta diferencia de 316 luxes no se considera relevante si se tiene en cuenta que el nivel de luminosidad toma como referencia una superficie en la que el flujo luminoso se distribuye uniformemente.

\subsection{Comparativa}

El método propuesto para comparar los resultados de los algoritmos de las técnicas de
Además, en la figura 4, se muestra la variación del nivel de luminosidad obtenida al grabar los detección de personas se basa en la determinación del número de aciertos en las detecciones, así como el número de falsos positivos y falsos negativo(Guerrero Balaguera, 2016). Estos valores se determinan para cada una de las tres técnicas en estudio, tanto para las dos alturas de localización como para los dos ángulos de inclinación del dispositivo de captura de vídeo, así como el nivel de luminosidad medio en el momento de la captura de cada vídeo, a fin de determinar el porcentaje de precisión de cada una de las técnicas.

\section{RESULTADOS}

En la tabla 4, se muestra la comparación de las técnicas de sustracción de fondo, detectores en cascada y flujo óptico, respecto al número de detecciones realizadas. En ella, se muestra la precisión de cada una de las técnicas teniendo en cuenta el número de falsos positivos y falsos negativos, en relación al número de personas que intervienen en el cuadro de video en análisis.

\begin{tabular}{c|c|c|c|c|c|c|c}
\hline \multicolumn{2}{c}{ Tabla 4. Comparativa de las técnicas de deteción de personas } \\
$\begin{array}{c}\text { Técnica } \\
\begin{array}{c}\text { Sustracción } \\
\text { de fondo }\end{array}\end{array}$ & Altura & $\begin{array}{c}\text { Ángulo de } \\
\text { inclinación }\end{array}$ & $\begin{array}{c}\text { Número } \\
\text { real de } \\
\text { personas }\end{array}$ & $\begin{array}{c}\text { Detecciones } \\
\text { realizadas }\end{array}$ & $\begin{array}{c}\text { Falsos } \\
\text { positivos }\end{array}$ & $\begin{array}{c}\text { Falsos } \\
\text { negativos }\end{array}$ & Exactitud \\
$\begin{array}{c}\text { Sustracción } \\
\text { de fondo }\end{array}$ & $4 \mathrm{~m}$ & $90^{\circ}$ & 60 & 59 & 0 & 1 & $98.34 \%$ \\
$\begin{array}{c}\text { Sustracción } \\
\text { de fondo }\end{array}$ & $8 \mathrm{~m}$ & $50^{\circ}$ & 60 & 55 & 2 & 3 & $94.73 \%$ \\
$\begin{array}{c}\text { Sustracción } \\
\text { de fondo }\end{array}$ & $8 \mathrm{~m}$ & $90^{\circ}$ & 60 & 54 & 1 & 5 & $91.5 \%$ \\
$\begin{array}{c}\text { Detectores } \\
\text { en cascada }\end{array}$ & $4 \mathrm{~m}$ & $50^{\circ}$ & 60 & 57 & 1 & 4 & $93.12 \%$ \\
$\begin{array}{c}\text { Detectores } \\
\text { en cascada }\end{array}$ & $4 \mathrm{~m}$ & $90^{\circ}$ & 60 & 54 & 0 & 6 & $90 \%$ \\
$\begin{array}{c}\text { Detectores } \\
\text { en cascada }\end{array}$ & $8 \mathrm{~m}$ & $50^{\circ}$ & 60 & 55 & 2 & 7 & $87.86 \%$ \\
$\begin{array}{c}\text { Detectores } \\
\text { en cascada }\end{array}$ & $8 \mathrm{~m}$ & $90^{\circ}$ & 60 & 54 & 1 & 7 & $88.21 \%$ \\
$\begin{array}{c}\text { Flujo } \\
\text { optico }\end{array}$ & $4 \mathrm{~m}$ & $50^{\circ}$ & 60 & 45 & 1 & 16 & $73.28 \%$ \\
$\begin{array}{c}\text { Flujo } \\
\text { optico }\end{array}$ & $4 \mathrm{~m}$ & $90^{\circ}$ & 60 & 48 & 1 & 13 & $78.26 \%$ \\
$\begin{array}{c}\text { Flujo } \\
\text { optico }\end{array}$ & $8 \mathrm{~m}$ & $50^{\circ}$ & 60 & 52 & 3 & 11 & $81 \%$ \\
$\begin{array}{c}\text { Flujo } \\
\text { optico }\end{array}$ & $8 \mathrm{~m}$ & $90^{\circ}$ & 60 & 53 & 1 & 8 & $86.56 \%$ \\
\hline
\end{tabular}


La técnica de sustracción de fondo aplicada con la cámara de video ubicada a 4 metros de altura con ángulo de inclinación de $50^{\circ}$ presenta mejores resultados en comparación con las demás configuraciones de ubicación, puesto que, de las 60 personas presentes en el video analizado, logró la detección de 59 de ellas. Para este caso, no se registra ningún caso de falso positivo, garantizando que cada una de las detecciones efectuadas correspondan a personas. Por otra parte, la técnica de flujo óptico probada a una altura de 4 metros con ángulo de inclinación de $50^{\circ}$ presenta los resultados con el mayor número de desaciertos, ya que, de las 60 personas a detectar, se realizó la detección correcta solo de 44 de ellas, sumado a que una de las detecciones correspondió a un falso positivo.

No obstante, en términos generales, las 3 técnicas de detección de personas presentan mejorías en el número de aciertos en las detecciones al ser implementadas en alturas de 8 metros con ángulo de inclinación del dispositivo de captura de video de $90^{\circ}$, ya que presentan una exactitud medida de $88.76 \%$, seguida con $88.25 \%$ de la implementación a una altura de 4 metros con $50^{\circ}$ de inclinación, por su parte, la implementación a 4 metros de altura con inclinación de $90^{\circ}$ y a 8 metros de altura con $50^{\circ}$ de inclinación, presentan rendimiento promedios de $87.66 \%$ y $87.62 \%$.

\section{CONCLUSIONES}

Al caracterizar el espacio en el cual se ubicará el dispositivo de captura, se aumenta el porcentaje de aciertos en las detecciones. Para la técnica de sustracción de fondo que fue la de mejor rendimiento, permitió pasar de 91,5\% de exactitud al estar ubicada a 8 metros de altura y con inclinación de $90^{\circ}$, a $98.34 \%$ si se ubica a 4 metros de altura con inclinación de $50^{\circ}$.

Las técnicas de sustracción de fondo y detectores en cascada al tener en promedio el mayor porcentaje de aciertos en las detecciones con $94.64 \%$ y $89.79 \%$ respectivamente, pueden ser aplicadas en procesos de video vigilancia y conteo automático de personas. Por su parte, la técnica de flujo óptico al no tener un porcentaje promedio de acierto superior a $80 \%$, no es recomendable su uso en procesos de detección de personas en tiempo real.

\section{REFERENCIAS}

Díaz, A. (2012). Cámaras de medición. Revista Universidad EAFIT, 26, 7-16. Retrieved from

http://publicaciones.eafit.edu.co/index.php/re vista-universidad-eafit/article/view/1528

Franco, C. E., Ospina, C. T., Cuevas, E. S., \& Capacho, D. V. (2017). RECONOCIMIENTO FACIAL BASADO EN EIGENFACES, LBHP Y FISHERFACES EN LA BEAGLEBOARDxM. Revista Colombiana De Tecnologias De Avanzada (Rcta), 2(26). https://doi.org/10.24054/16927257.v26.n26.2 015.2387

García S, I., \& Caranqui S, V. (2015). La visión artificial y los campos de aplicación. Tierra Infinita, 1, 94-103. https://doi.org/2631-2921

Guerrero Balaguera, J. D. (2016). Algoritmos De Procesamiento De Imágenes Y Redes Neuronales Artificiales Para El Reconocimiento De La Lengua De Señas Colombiana (Lsc) Image Processing Algorithms and Artificial Neural Networks To Recognition of Colombian Sign Language (Lsc). Revista Colombia de Tecnologías de Avanzada RCTA, 1-8.

Hernández Tolosa C, Contreras Eugenio B, Torres Sánchez C. (2016). Desarrollo de libros electrónicos: "taller pedagógico". Revista Tecnologías de Avanzada, ISSN: 1692-7257

Jeon, E. S., Choi, J. S., Lee, J. H., Shin, K. Y., Kim, Y. G., Le, T. T., \& Park, K. R. (2015). Human detection based on the generation of a background image by using a far-infrared light camera. Sensors (Switzerland), 15(3), 6763-6788. https://doi.org/10.3390/s150306763

Kajabad, E. N., \& Ivanov, S. V. (2019). People Detection and Finding Attractive Areas by the use of Movement Detection Analysis and Deep Learning Approach. Procedia Computer Science, 156, 327-337. https://doi.org/10.1016/j.procs.2019.08.209

Leo, M., Medioni, G., Trivedi, M., Kanade, T., \& Farinella, G. M. (2017). Computer vision for assistive technologies. Computer Vision and Image Understanding, 154, 1-15. https://doi.org/10.1016/j.cviu.2016.09.001

Mahkonen, K., Virtanen, T., \& Kämäräinen, J. (2018). Cascade of Boolean detector combinations. Eurasip Journal on Image and Video Processing, 2018(1). https://doi.org/10.1186/s13640-018-0303-9 
Min, Q., \& Huang, Y. (2016). Motion detection using binocular image flow in dynamic scenes. Eurasip Journal on Advances in Signal Processing, 2016(1). https://doi.org/10.1186/s13634-016-0349-8

Moctezuma-Ochoa, D. A. (2016). Reidentificación de personas a través de sus características soft-biométricas en un entorno multi-cámara de video-vigilancia. Ingeniería, Investigación y Tecnología, 17(2), 257-271. https://doi.org/10.1016/j.riit.2016.06.010

Paul, M., Haque, S. M. E., \& Chakraborty, S. (2013). Human detection in surveillance videos and its applications - a review. Eurasip Journal on Advances in Signal Processing, 2013(1), 1-16. https://doi.org/10.1186/1687-6180-2013-176

Pérez, J, Castro, J (2018). LRS1: un robot social de bajo costo para la asignatura "Programación 1". Revista Tecnologías de Avanzada, ISSN: 1692-7257

Setjo, C. H., Achmad, B., \& Faridah. (2017). Thermal image human detection using Haarcascade classifier. Proceedings - 2017 7th International Annual Engineering Seminar, InAES 2017, (January 2020). https://doi.org/10.1109/INAES.2017.806855 4

Sharmin, N., \& Brad, R. (2012). Optimal filter estimation for Lucas-Kanade optical flow. Sensors (Switzerland), 12(9), 12694-12709. https://doi.org/10.3390/s120912694

Sobral, A., \& Vacavant, A. (2014). A comprehensive review of background subtraction algorithms evaluated with synthetic and real videos. Computer Vision and Image Understanding, 122, 4-21. https://doi.org/10.1016/j.cviu.2013.12.005

Thacker, N. A., Clark, A. F., Barron, J. L., Ross Beveridge, J., Courtney, P., Crum, W. R., ... Clark, C. (2008). Performance characterization in computer vision: A guide to best practices. Computer Vision and Image Understanding, 109(3), 305-334. https://doi.org/10.1016/j.cviu.2007.04.006

Vidalia, H., Sandoval, G., Rogelio, L., Gill, V., Curiel, A. A., Alberto, F., ... L, A. M. (2019). Una estrategia didáctica interactiva e interdisciplinar para el proceso de aprendizaje. Técnica Estenopeica; Repreproduccion Visual de La Realidad., l(2), 9-27. https://doi.org/10.22517/25393812.21971 